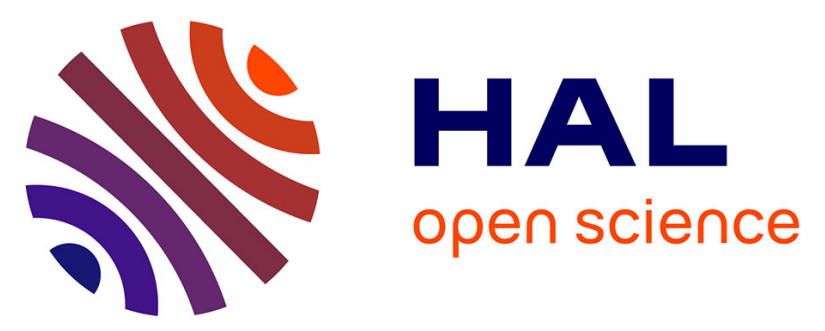

\title{
APPORT DE LA VITESSE DU SON À LA CONNAISSANCE DE LA COMPACTION DES SOLS. Étude des premières phases de tassement des sols sous-marins
}

P. Morlier, C. Viguier, P. Guillaume

\section{To cite this version:}

P. Morlier, C. Viguier, P. Guillaume. APPORT DE LA VITESSE DU SON À LA CONNAISSANCE DE LA COMPACTION DES SOLS. Étude des premières phases de tassement des sols sous-marins. Journal de Physique IV Proceedings, 1992, 02 (C1), pp.C1-977-C1-980. 10.1051/jp4:19921214 • jpa00251181

\section{HAL Id: jpa-00251181 \\ https://hal.science/jpa-00251181}

Submitted on 1 Jan 1992

HAL is a multi-disciplinary open access archive for the deposit and dissemination of scientific research documents, whether they are published or not. The documents may come from teaching and research institutions in France or abroad, or from public or private research centers.
L'archive ouverte pluridisciplinaire HAL, est destinée au dépôt et à la diffusion de documents scientifiques de niveau recherche, publiés ou non, émanant des établissements d'enseignement et de recherche français ou étrangers, des laboratoires publics ou privés. 
APPORT de LA VITESSE du SON À LA CONNAISSANCE dE LA COMPACTION DES SOLS. Etude des premières phases de tassement des sols sous-marins

\author{
P. MORLIER, C. VIGUIER et P. GUILLAUME \\ Laboratoire d'Etudes et de Recherches en Géotechniques et Géophysique Appliquée, Université de \\ Bordeaux I, 351 Cours de la Libération, F-33405 Talence cedex, France
}

\begin{abstract}
An experimental study which measure the sound velocity during the settling of marine sediments is presented. In the first step the sound velocity slightly decrease during decantation stage, in the second step it increases with loading and porosity reduction during compaction stage (WOOD (1941) and NOBES (1989) models). The follow of the process shows the progressive rearrangement of particules structure and the acquisition of rigidity appears at a threshold porosity (about 0,70 ). Acoustical behaviour of argileous and calcareous sediments mainly differs owing to grain-size distribution which control the particles reorganization.
\end{abstract}

\title{
Résumé
}

La vitesse du son est mesurée dans des sédiments marins lors d'une étude expérimentale du tassement. Durant la phase de sédimentation, la vitesse du son diminue légèrement, puis lors de la phase de compaction elle s'accroît avec la charge et la réduction de porosité (modèles de WOOD (1941) et de NOBES (1989). Le suivi du phénomène met en évidence le réarrangement graduel de la structure des particules et l'apparition de la rigidité pour une porosité seuil (environ 0,70). La différence de comportement acoustique entre les matériaux argileux et carbonatés résulte essentiellement de la granulométrie qui contrôle la réorganisation des grains

\section{INTRODUCTION}

L'étude géotechnique et sédimentologique de sédiments sous-marins nous ont amenés à étudier expérimentalement, à l'aide de la vitesse du son, la sédimentation et le début de la compaction des sols argileux, argileux carbonatés et carbonatés. Le rapprochement avec l'essai oedométrique classique réalisé en même temps contrôle la validité des résultats et souligne l'originalité de la démarche.

\section{Dispositif expérimental}

Sur la surface interne d'une cellule oedométrique de $50 \mathrm{~mm}$ de diamètre et $20 \mathrm{~mm}$ de hauteur, deux capteurs ultrasoniques sont diamétralement opposés dans un plan horizontal (fig. 1). Les transducteurs utilisés sont des céramiques piézoélectriques cylindriques de fréquence $300 \mathrm{kHz}$ vibrant dans le sens de l'épaisseur. La distance entre les deux transducteurs étant constante on peut déterminer à chaque instant la célérité des ondes longitudinales à travers le matériau La cellule est surmontée d'un tube en plexiglass de 50 cm de haut pour l'essai de sédimentation.

Lors de la phase de sédimentation, l'appareil est rempli d'eau de salinité $35 \%$ pour l'étalonnage des mesures de vitesse du son. Puis les particules sont mises en dispersion, la hauteur du floculat ainsi que la célérité des ondes longitudinales à la base de l'éprouvette sont mesurées en fonction du temps (Fig. 2). L'évaluation du tassement se fait par mesure de la hauteur relative de la solution (MIGNIOT, 1968, fig. 2): 

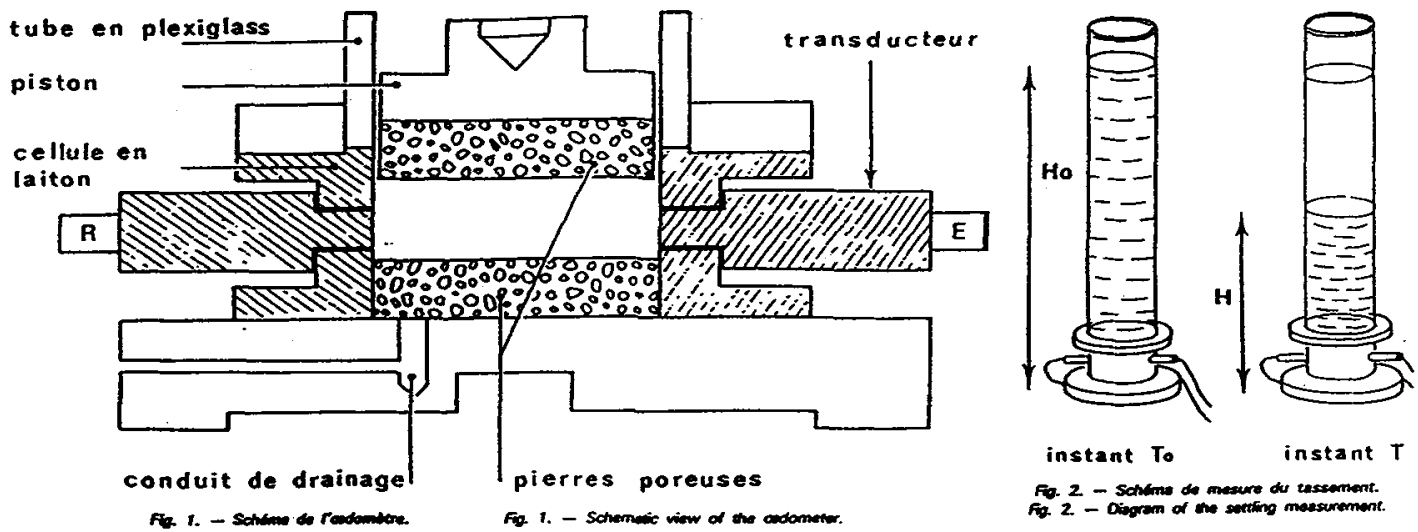

L'essai oedométrique se déroule ensuite sur le culot suivant deux cycles chargedécharge $(\Delta \sigma / \sigma=1$, durée de charge $24 \mathrm{~h}$, contrainte maximale $2 \mathrm{MPa})$.

\section{Sols étudiés}

Les matériaux étudiés sont des échantillons naturels de granulométrie et de teneur en carbonate variées provenant du domaine Caraibe ainsi que des mélanges réalisés à partir de sédiments argileux et de particules carbonatées. Ils se répartissent en trois classes : sables carbonatés $\left(\mathrm{CaCO}_{3} \approx 70 \%\right)$ : $\mathrm{SC1}$; silts sableux carbonatés $\left(\mathrm{CaCO}_{3} \approx 60 \%\right)$ : SS1-SS2 ; argiles silteuses $\left(\mathrm{CaCO}_{3}: 25-35 \%\right)$ : AS1-AS3, qui correspondent à peu près à l'éventail des sols compressibles.

En première approche les carbonates interviennent comme éléments granulométriques des classes "sables et silts" essentiellement. Durant la phase de sédimentation, la hauteur relative $\mathrm{H} / \mathrm{H}_{0}$ en fonction du logarithme du temps (fig. 3) se traduit par une courbe sigmoïde classique, plus ou moins accentuée selon la granulométrie du matériau et sa concentration initiale [2]. Le tassement par décantation est complété par l'essai oedométrique. Les deux comportements à la compaction représentés sont typiques de la granulométrie et de la nature des deux catégories de matériaux fins testés (fig. 4). Les échantillons argileux (AS4) possédent un comportement. de type "normal" et celui des silts carbonatés (SS3) de type "fragile" caractérisé par la rupture des particules [1]. Ils ont été choisis pour l'étude acoustique du tassement, le second constituant en quelque sorte une limite des sols compressibles.
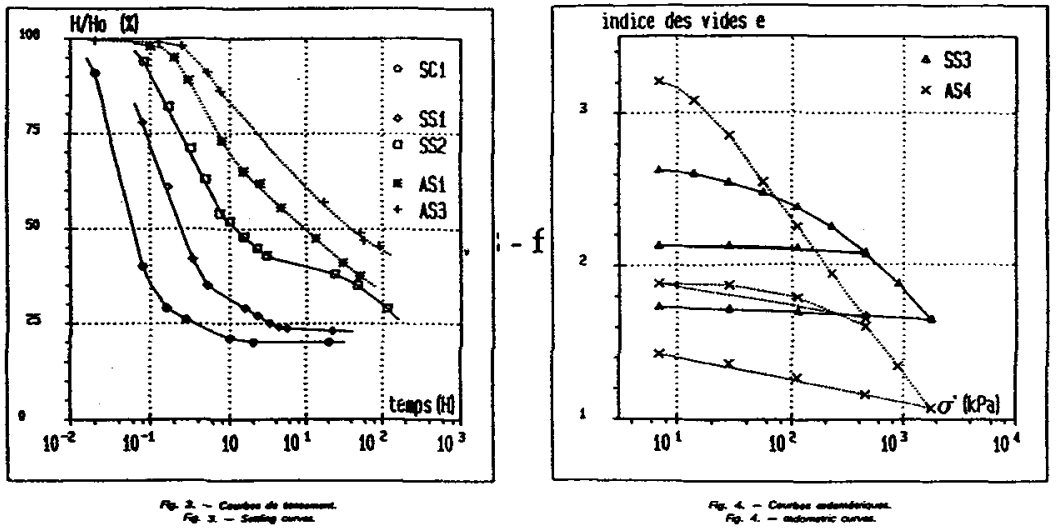

3. Tassement et vitesse du son

Dans les sédiments sableux, presque exclusivement formés de tests de foraminiferes, la vitesse du son est de l'ordre de $1525 \mathrm{~m} / \mathrm{s}$, légèrement supérieure à celle dans l'eau de mer : les matériaux de type sableux acquièrent une structure rigide dès leur dépôt. 
Pour les échantillons silteux ou argileux, une légère diminution de la vitesse du son apparaît lors de la sédimentation (SS2) en trois phases (fig. 5a). Vitesse du son voisine de celle de l'eau de mer $\left(V_{L}=1500 \mathrm{~m} / \mathrm{s}\right)$ : propagation des ondes dans l'eau; Vitesse du son diminue : complexe eau particule entrave la propagation ; Vitesse du son stabilisée à 1460$1480 \mathrm{~m} / \mathrm{s}$ : dépôt stabilisé.

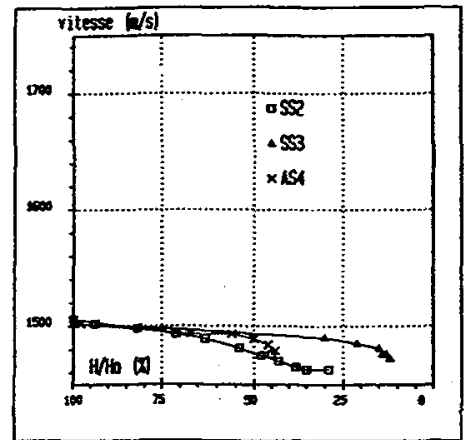

Fig. 5. - Vitesseo du son in tonction du trassememt. F. Arece de escimintintion.

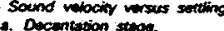

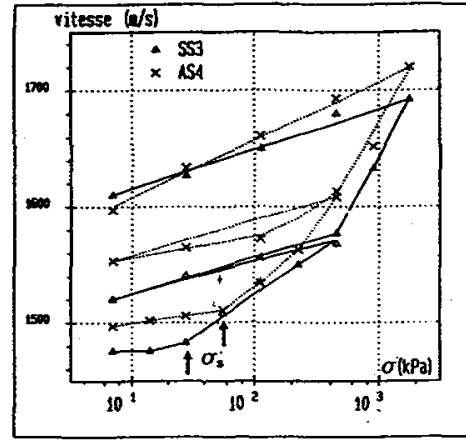

F. 5. - Vhesse du son an fonction du tassement. Fig. 5. - Sound we compity versus setting.

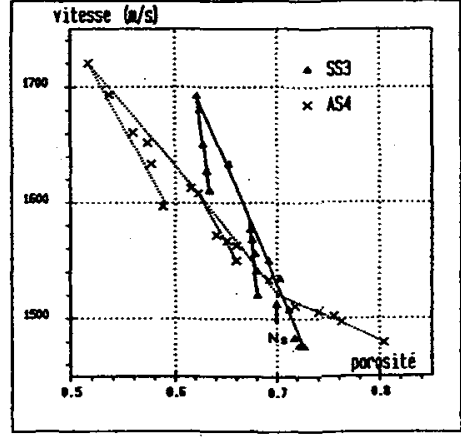

F5. 6. - Vitose du son on tonction de to parasite. Fig. 6. - sound velocity versus porasing.

Sous charge croissante, la célérité des ondes acoustiques s'accroît. La contrainte effective contrôle le rapprochement des particules. L'allure des courbes vitesse-contrainte rappelle celle des courbes oedométriques (fig. $5 \mathrm{~b}$ et fig. 4) ; Toutefois les courbes "vitesse" des silts carbonatés (SS3) et des argiles (AS4) sont très proches et moins "typiques" des sédiments que les courbes oedométriques. Elles se décomposent en trois parties (fig . 5) : un palier initial à faible variation de la vitesse du son $\left(V_{L} \approx 1500 \mathrm{~m} / \mathrm{s}\right)$; à partir d'une certaine valeur de la contrainte $\left(\sigma_{S}^{\prime}\right)$, la vitesse s'accroît nettement ; $\sigma^{\prime}$ s matérialise un changement d'état du sédiment; pour les charges plus élevées, la croissance des vitesses devirent plus rapide et varie linéairement avec celle de la contrainte.

Les deux phases successives de l'évolution de la vitesse des ondes dans l'échantillon argileux AS4 marquent deux phénomènes distincts qui se produisent simultanément mais avec prépondérance de l'un puis de l'autre : réarrangement des particules, puis rapprochement par expulsion de l'eau. Pour les silts carbonatés SS3, le passage entre ces deux phases est progressif et traduit des processus de réorganisation des grains plus importants. Les valeurs de $\sigma^{\prime}$, correspondant au début du réarrangement, sont comprises entre 15 et $80 \mathrm{kPa}$, suivant la nature du sédiment. Nous retrouvons là, clairement marqué, les résultats expérimentaux d'une orientation préférentielle des particules à partir d'une surcharge de $100 \mathrm{kPa}[3,4,5]$.

Lors de l'accroissement de la contraintes, nous suivons la diminution des vides par la variation de la porosité (fig. 6). La courbe de chargement de l'échantillon argileux (AS4) fait apparaître deux segments linéaires : pour les fortes porosités la vitesse augmente très légèrement quand la porosité diminue, pour des plus faibles porosités la vitesse s'accroît fortement. La limite entre deux tronçons est une valeur seuil de porosité $\mathbf{n}_{\mathrm{s}}$ caractéristique du matériau, correspondant à la contrainte seuil déterminée précédemment (Pour SS3 la porosité seuil est assimilable à la porosité initiale). Cette valeur est à comparer aux valeurs classiques de changement $d^{\prime}$ état : limite de liquidité d'Attenberg, transition vasefloculat habituellement prise pour le minimum de la courbe de WOOD $[6,3,7]$.

La vitesse du son permet d'affiner la détermination du passage des comportements liquide à plastique et de préciser l'acquisition d'une structure rigide.

\section{Rapprochement à la théorie}

Les sédiments que nous avons analysés possédant une porosité élevée, les théories concernant les suspensions et les agrégats granulaires sont mieux adaptées que celles des milieux poreux.

Dans les suspensions et émulsions, la vitesse du son évolue selon une loi simple de mélange à deux composantes [7], (fig. 7).

Mais d'une manière générale, les mesures de célérité des ondes sur des sédiments naturels donnent des valeurs supérieures à celles de WOOD $[8,9,10,11,12]$. 
Des théories sur les milieux poreux saturés, nous retiendrons le modèle de WYLLIE et al. [15] qui prend en compte le comportement propre à chaque phase, liquide ou solide.

Nous retiendrons également la combinaison des modèles de WOOD et de WYLLIE qui considère le fluide comme une suspension et les particules comme des matériaux poreux, de NOBES [14], (fig. 7).
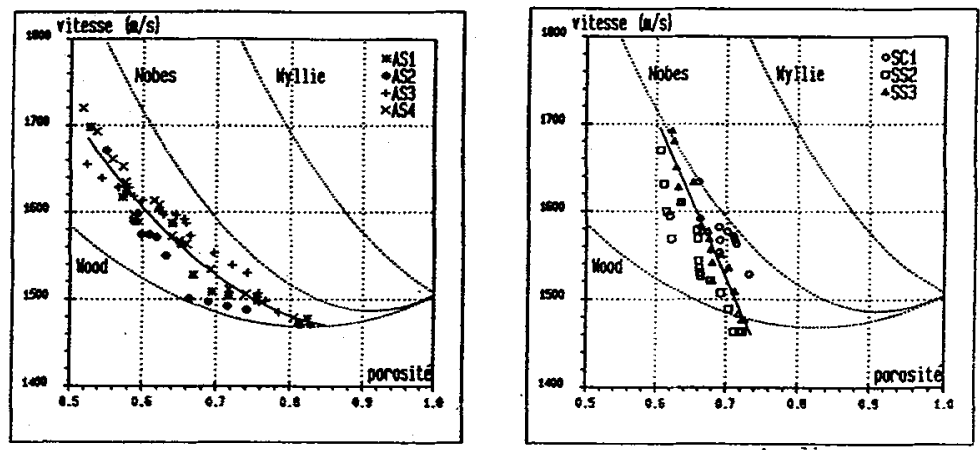

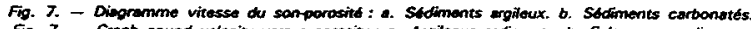

Les échantillons argileux se localisent entre les courbes des modèles de WOOD et de NOBES, ces sédiments présentent un comportement typique de suspensions possédant déjà une certaine rigidité (fig. $7 \mathrm{a}$ ). Par contre l'évolution des silts carbonatés est différente et leur comportement se rapproche de celui des milieux poreux du modèle de WYLLIE (fig. $7 b)$. Bien que la vitesse du son dans les carbonates et les silicates soit plus élevée que dans les argiles, la composition chimique seule ne permet pas d'expliquer la différence de comportement acoustique entre les deux types de matériaux. Linfluence de la granulométrie transparaît $[8,9,10]$.

\section{CONCLUSION}

La mesure de la vitesse du son pendant les premiers stades du tassement expérimental des sols-sous-marins étudiés permet de suivre avec précision les changements d'états des matériaux, cohésion puis rigidité, qui débutent pour des contraintes inférieures à $100 \mathrm{kPa}$.

L'étude confirme en outre la sensibilité des ondes sonores aux porosités : diminution de la vitesse du son pendant la sédimentation puis augmentation lors de la compaction (modèle de WOOD et de NOBES).

\section{BIBLIOGRAPHIE}

[1] GUILLAUME P. - Thèse de l'Université de Bordeaux I, 1990, 258 p.

[2] MIGNIOT C. - La Houille Blanche, $\mathrm{n}^{\circ}$ spécial 7, 1968, 591-620.

[3] O'BRIEN D.C. - J. Acoust. Soc. An.,1, 1989, 291-294.

[4] MARTIN R.T. - North Am. Clay Miner. Conf., 14th, Berkaley, 1965, 21 p.

[5] RIEKE H.H., CHILINGARIAN G.V. - Developments in Sedimentology $n^{\bullet} 16$. Elsevier, 1974, 424

[6] MIZIKOS J.P. - Thèse de Doct. ès Sciences, Nice, 1972, 203 p.

[7] WOOD A.B. - A text book of sound. G. Belle and sons, London 1941.

[8] GREFFARD J. - Cahier Océan., Paris, XXI, 2, 145-171.

[9] HAMILTON E.L. et al. - J. Sed. Pet., 52, 3, 1982, 733- 753.

[10] BACHMAN R.T. - J. Acoust. Soc. Am., 78, 22, 1985, 615-621.

[11] ORSI T.H., DONN D.A. - J. Acoust. Soc. Am. 88, 3, 1990, 1536-1542.

[12] LAUGHTON A.S. - Geophysics, 22, 2, 1957, 31 p.

[13] WYLLIE M.R., GARDNER G.H., GREGORY A.R. - Geophysics, 27, 1962, 569-589.

[14] NOBES D.C. - J. Acoust. Soc. Am., 86, 1, 1989, 291-294.

[15] MORLIER P., VIGUIER C., GUILLAUME P., - Rev. Franç. Geotech., 57, 1991, $49-57$. 